\title{
Biochemical and Structural Investigation of GnnA in the Lipopolysaccharide Biosynthesis Pathway of Acidithiobacillus ferrooxidans
}

Juthatip Manissorn ${ }^{a}$, Thassanai Sitthiyotha ${ }^{b}$, Jenny Rose E. Montalban ${ }^{c}$, Surasak Chunsrivirot ${ }^{b}$, Peerapat Thongnuek ${ }^{a}$, Kittikhun Wangkanont ${ }^{d, *}$

${ }^{a}$ Biomedical Engineering Research Center (BMERC) and Biomedical Engineering Program, Faculty of Engineering, Chulalongkorn University, Bangkok 10330 Thailand

${ }^{\mathrm{b}}$ Structural and Computational Biology Research Unit, Department of Biochemistry, Faculty of Science, Chulalongkorn University, Bangkok 10330 Thailand

${ }^{c}$ Department of Biochemistry, Faculty of Pharmacy, University of Santo Tomas, Manila 1008 Philippines

${ }^{\mathrm{d}}$ Center of Excellence for Molecular Biology and Genomics of Shrimp, and Molecular Crop Research Unit, Department of Biochemistry, Faculty of Science, Chulalongkorn University, Bangkok 10330 Thailand 




Figure S1. Initial rate of NADH formation from oxidation of UDP-GlcNAc by NAD ${ }^{+}$, both at 5 $\mathrm{mM}$, in different buffers with $1 \mu \mathrm{M} A f \mathrm{GnnA}$. Each buffer consisted of $50 \mathrm{mM}$ buffer salt at the indicated $\mathrm{pH}$ and $150 \mathrm{mM} \mathrm{NaCl}$. 


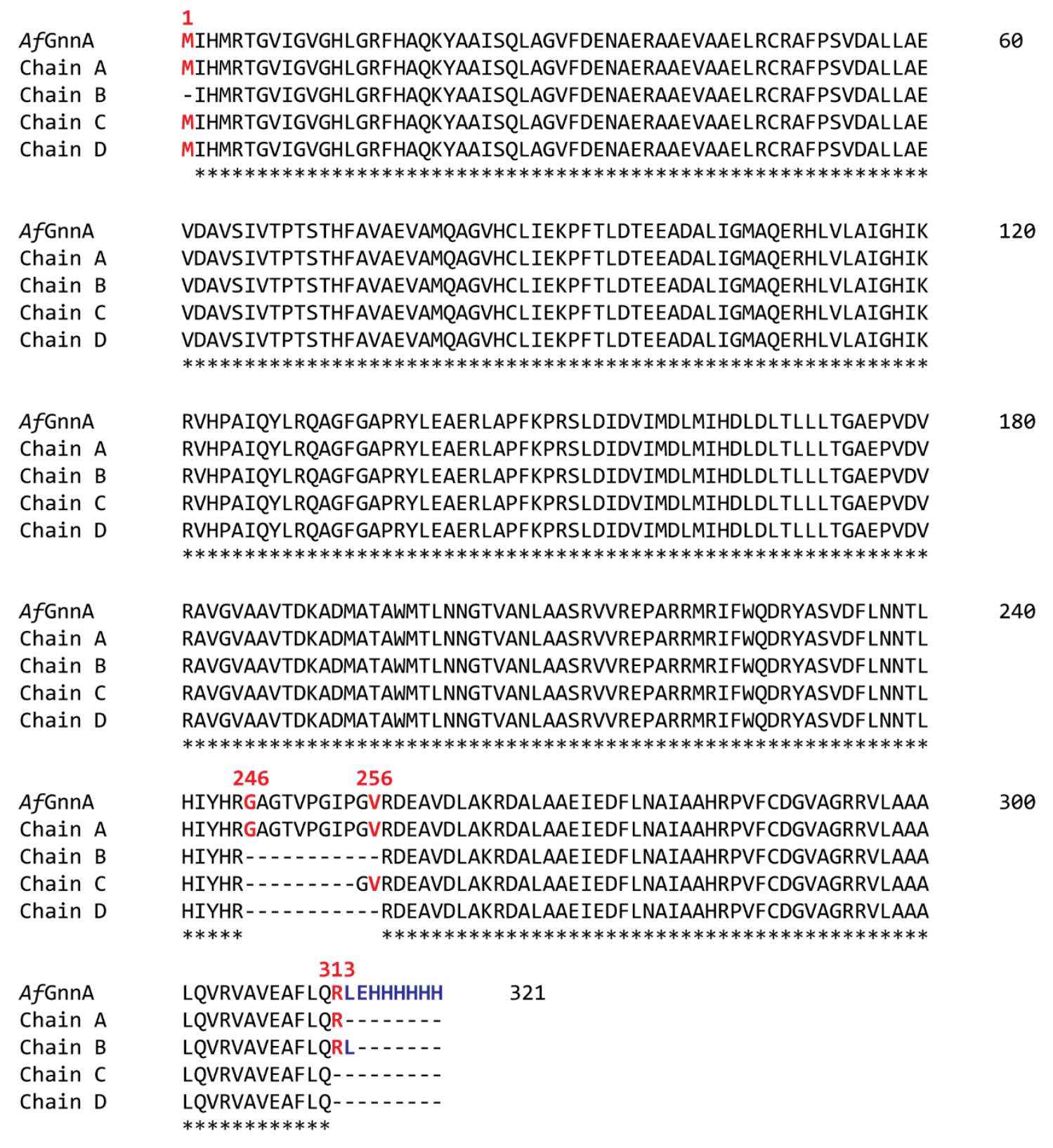

Figure S2. Alignment of the $A f \mathrm{Gnn} A$ amino acid sequence and the sequences from the monoclinic AfGnnA crystal structure (PDB ID 7BVJ). AfGnnA is 313 amino acids long. Residue near the boundaries are shown in red. The purification tag is shown in blue. Asterisks $\left(^{*}\right)$ indicate amino acids that are present in all chains. 


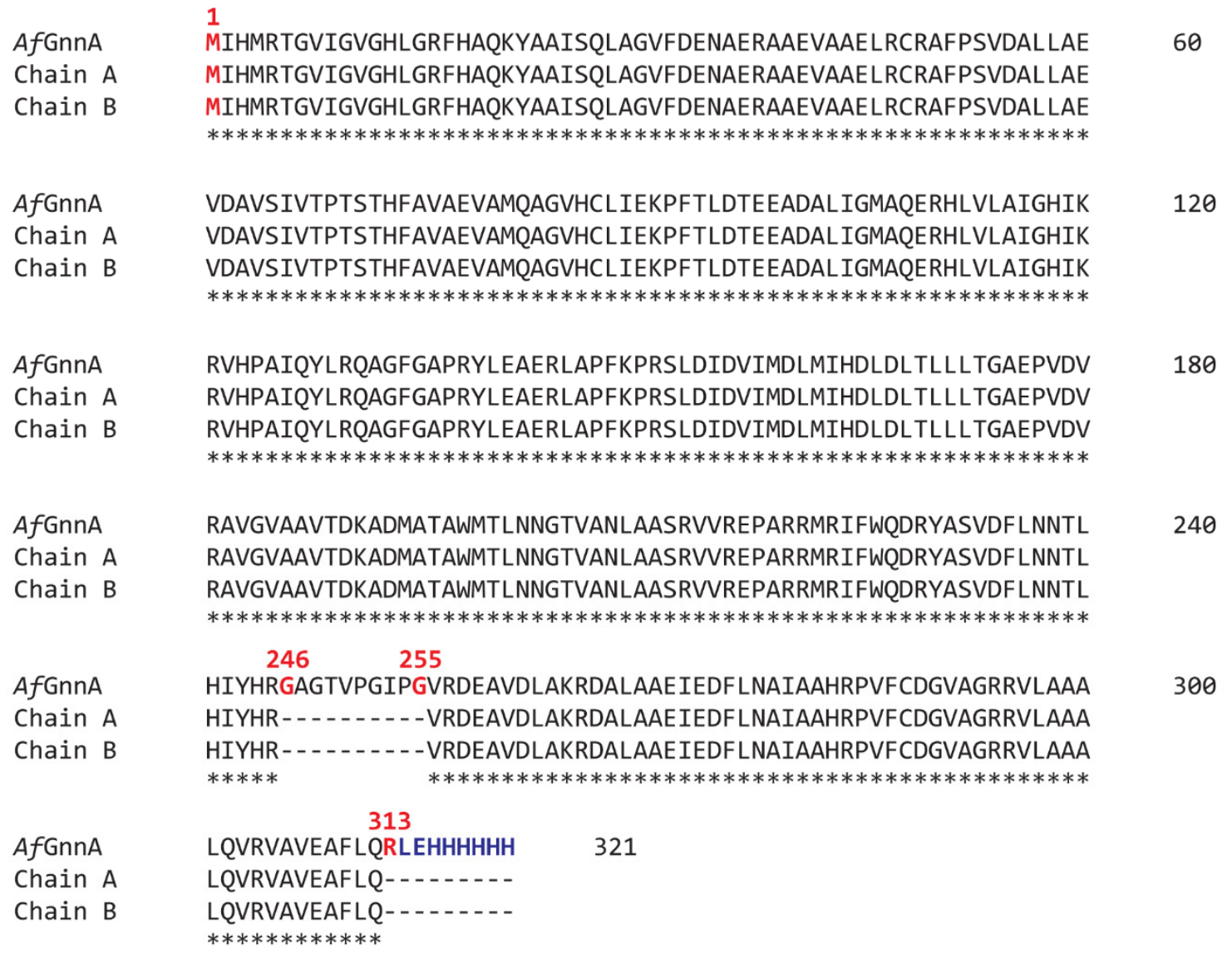

Figure S3. Alignment of the $A f \mathrm{GnnA}$ amino acid sequence and the sequences from the orthorhombic $A f \mathrm{GnnA}$ crystal structure (PDB ID 7BVK). Native $A f \mathrm{GnnA}$ is 313 amino acids long. Residue near the boundaries are shown in red. The purification tag is shown in blue. Asterisks $\left(^{*}\right)$ indicate amino acids that are present in all chains. 


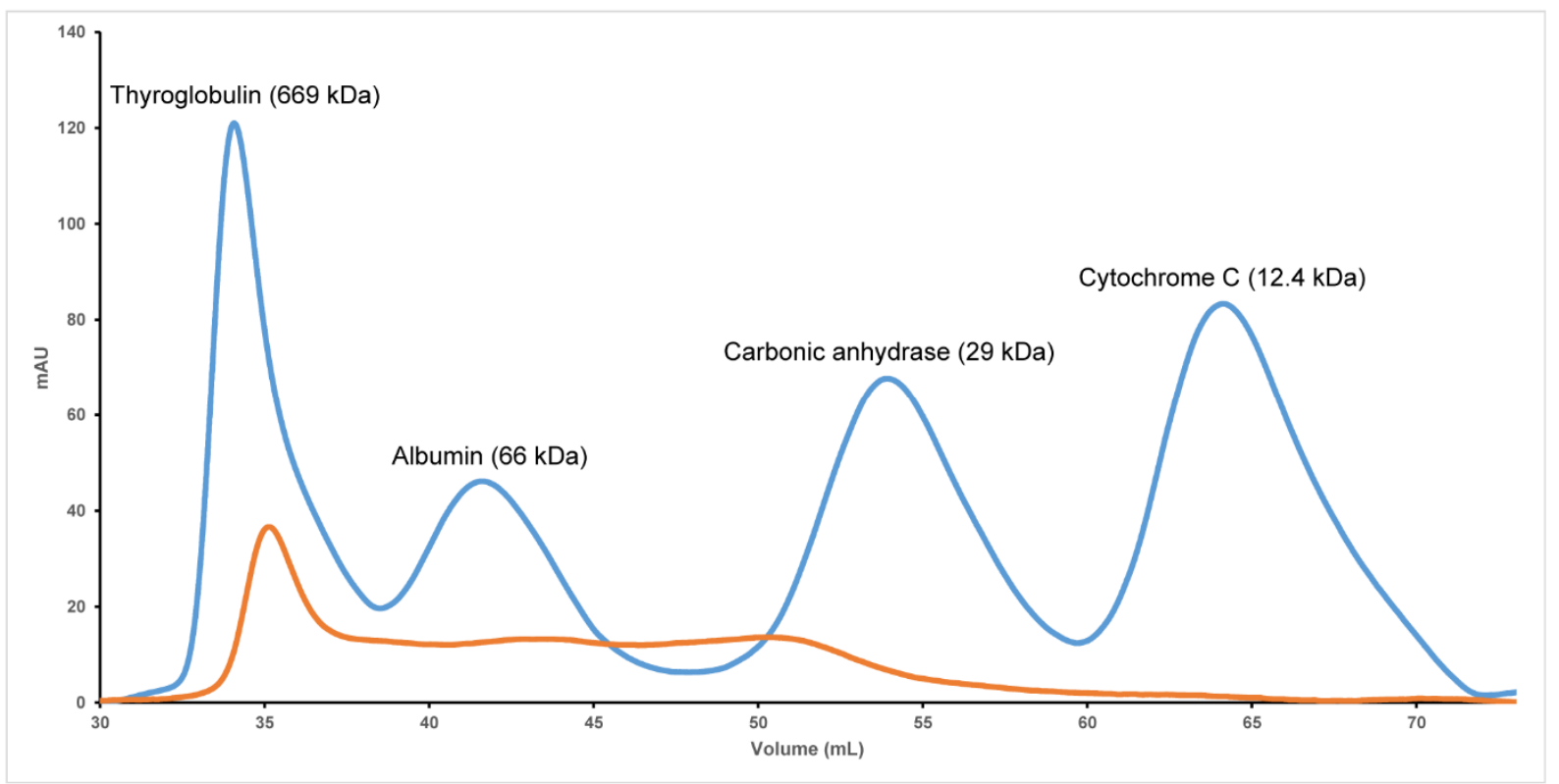

Figure S4. Gel filtration analysis of Ni-NTA purified $A f \mathrm{GnnA}$ on a HiPrep 16/60 Sephacryl S$100 \mathrm{HR}$ column (orange line). The expected molecular weight of $A f \mathrm{GnnA}$ is $35 \mathrm{kDa}$. Elution profile of molecular weight standards is overlaid for comparison (blue line). 


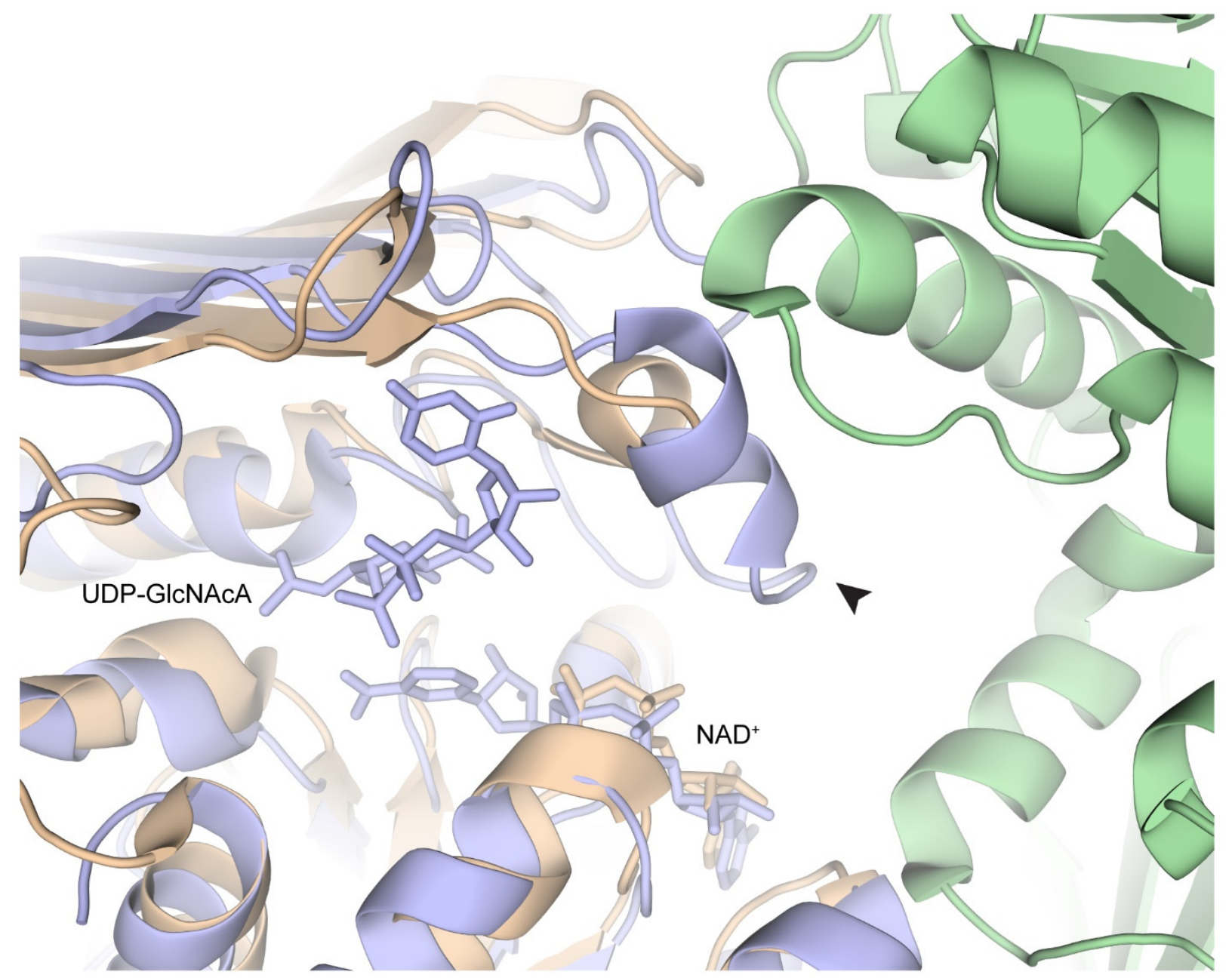

Figure S5. Superimposition of the crystal structure of Chromobactrium violaceum WlbA (PDB ID 3Q2I) with its UDP-GlcNAcA and $\mathrm{NAD}^{+}$ligands (light blue) onto the crystal structure of monoclinic $A f \mathrm{GnnA}$ (wheat and green). The arrow indicates the UDP-GlcNAcA binding region of WlbA that corresponds to the region used by $A f \mathrm{Gnn} A$ for crystal packing. 


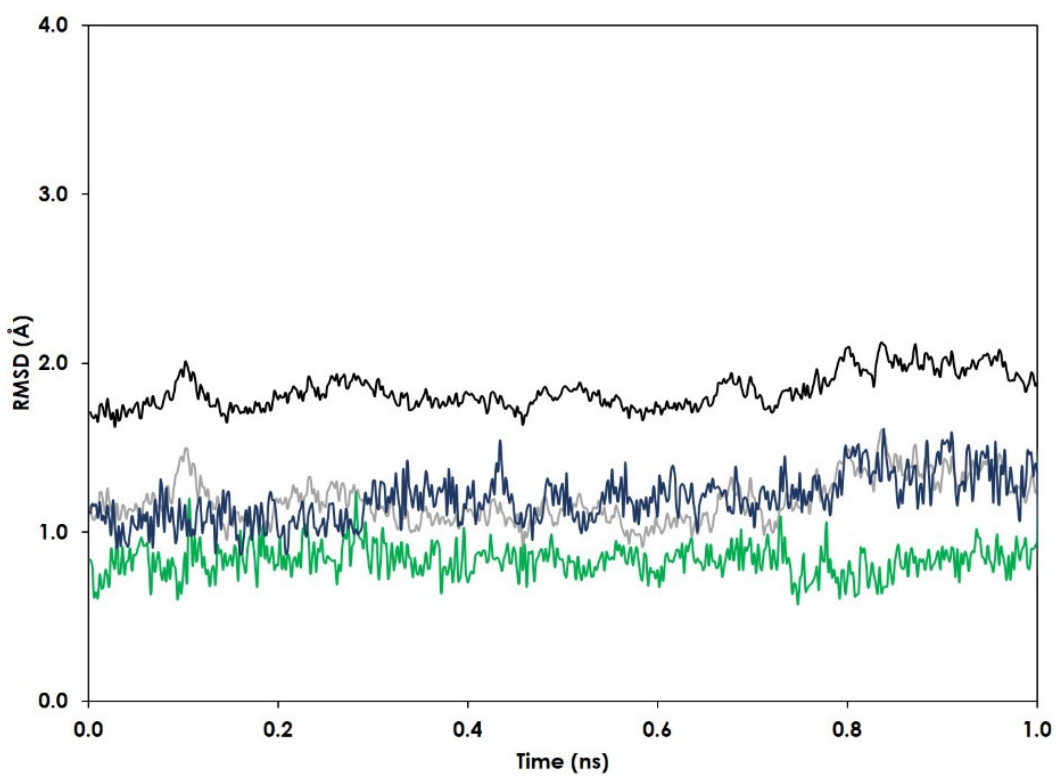

Figure S6. RMSD plots of the AfGnnA-ligands complex. The RMSD values of all atoms, backbone atoms, UDP-GlcNAc atoms and NAD ${ }^{+}$atoms are shown in black, grey, green and blue, respectively. 




Figure S7. The distances between atoms of ligands and important binding residues of the $A f G n n A-$ substrates complex during MD simulation(NAD @O 08 and D33@OD1 in purple, NAD $@ 04$ and D33@OD2 in blue, NAD @OO and H73@NE2 in green, NAD $@ 0$ O5 and K91@O in orange, UDP-GlcNAc@O8 and K91@NZ in red, UDP-GlcNAc@O12 and K91@NZ in cyan, UDPGlcNAc@H21 and H164@NE2 in grey and UDP-GlcNAc@H5 and NAD $@$ C13 in black). 
Table S1. The average distances between atoms of ligands and important binding residues of the AfGnnA-substrates complex

\begin{tabular}{|c|c|c|c|c|}
\hline \multicolumn{3}{|c|}{ atom-atom distance } & \multirow{2}{*}{$\begin{array}{c}\begin{array}{c}\text { average distance } \\
\text { from } \operatorname{MD}(\AA)\end{array} \\
2.7 \pm 0.1\end{array}$} & \multirow{2}{*}{$\begin{array}{l}\text { distance measured from the } \\
\text { minimized structure of the } \\
\text { last snapshot of MD }(\AA)\end{array}$} \\
\hline NAD $@ 08$ & --- & D33@OD1 & & \\
\hline NAD $+@ 04$ & --- & D33@OD2 & $2.7 \pm 0.1$ & 2.8 \\
\hline NAD+@O9 & --- & H73@NE2 & $3.5 \pm 0.3$ & 3.4 \\
\hline NAD+@O5 & --- & K91@O & $3.3 \pm 0.4$ & 3.2 \\
\hline UDP-GlcNAc@O8 & --- & K91@NZ & $3.1 \pm 0.3$ & 2.9 \\
\hline UDP-GlcNAc@O12 & --- & K91@NZ & $2.9 \pm 0.2$ & 2.9 \\
\hline UDP-GlcNAc@H21 & --- & H164@NE2 & $2.4 \pm 0.5$ & 2.2 \\
\hline UDP-GlcNAc@H5 & --- & NAD+@C13 & $3.3 \pm 0.4$ & 3.2 \\
\hline
\end{tabular}

